\title{
Tunable III-V-on-Si Laser with Resonant Photonic Molecule Mirrors
}

\author{
Guilherme F. M. de Rezende ${ }^{1,2, *}$, Newton C. Frateschi, ${ }^{1}$ and Gunther Roelkens ${ }^{2}$ \\ 1 “Gleb Wataghin" Physics Institute, University of Campinas, 13083-859 Campinas, SP, Brazil \\ ${ }^{2}$ Photonics Research Group, INTEC, Ghent University-imec 9052 Ghent Belgium \\ *rezendeg@ifi.unicamp.br
}

We propose, fabricate and characterize a novel III-V-on-Si laser. Resonant mirrors are realized by tailoring supermodes of coupled microrings. A threshold of $40 \mathrm{~mA}$, series resistance of $10 \Omega$ and SMSR of $40 \mathrm{~dB}$ is reported.

OCIS codes: $140.5960,230.4555,140.3600$

\section{Introduction and Discussion}

Heterogeneous integration of III-V materials on top of Si waveguides has become one of the most efficient methods for incorporating semiconductor lasers onto a Si photonic platform [1]. The maturing of different techniques for III-V-on-Si laser integration, e.g. BCB adhesive bonding [2], direct bonding [3] and transfer printing [4], has led to a myriad of laser devices relying on both high optical gain of III-V materials and low-loss, high index contrast and small footprint SOI waveguide circuits. The use of Si microring resonators for modal filtering and feedback to form a laser cavity has been demonstrated in several forms, see e.g. [2].

Here we present a novel design of a ring resonator based III-V-on-Si laser, where each mirror is realized by a system of coupled resonators composed by an outer ring coupled to two embedded rings mutually coupled, here called a photonic molecule. Figure 1(a) shows a sketch of the proposed idea. When all rings are on resonance, the mutual coupling of this odd number of resonators results in standing wave supermodes of the molecule due to the breaking of degeneracy between clockwise (CW) and counterclockwise (CCW) bare modes of the microrings [5]. If any of the rings is tuned out of resonance or if a different resonant order is chosen (in which case the Vernier effect forces the detuning between cavities), this phenomenon disappears. Seen from a bus waveguide coupled to the outer ring, the breaking of the degeneracy appears as an induced reflection which can be tailored according to a balance between coupling strength and detuning (Figure 1(c) and (d)).

(a)

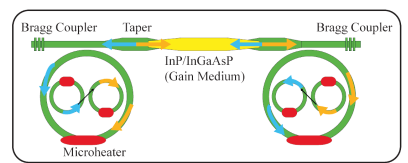

(b)

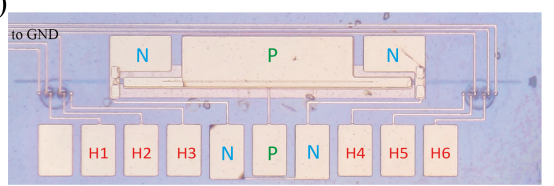

(c)

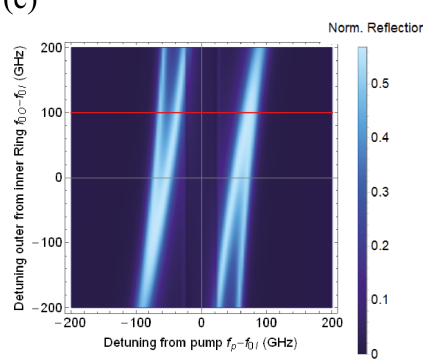

(d)

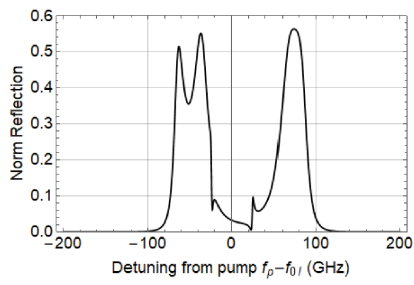

Fig. 1: (a) Schematics of the proposed laser structure with a pair of photonic molecule mirrors. Light propagating in $\mathrm{CW}$ direction (orange) is forced to couple to $\mathrm{CCW}$ direction (blue). The gain medium (yellow) in between the mirrors is bonded on the silicon waveguide circuit and tapers are used for coupling to the Si waveguide structure. (b) Micrograph of the fabricated device, with contact pads depicted: H1 to H6: microheaters pads; N: N-contact and P: P-contact of diode laser. All heaters share a common ground pad (not shown). (c) and (d) Simulated reflection for the photonic molecule around the maximum degeneracy. In (c), each horizontal line (e. g. red line) represents a reflection spectrum of a molecule for a given detuning between the outer ring and degenerated inner rings.

The device fabrication is based on DVS-BCB adhesive bonding of an InP $1550 \mathrm{~nm}$ amplifier stack on a 400 $\mathrm{nm}$ SOI e-beam patterned wafer. The detailed fabrication is reported in [2]. Optical coupling between the III-V amplifier and the silicon photonic integrated circuit is realized by means of a double inverted taper. Figure 1 (b) shows a typical fabricated device where each ring out of the photonic molecule has an integrated TiAu microheater for tunability. Gold pads allow biasing both the amplifier and the microheaters. 
Laser basic characterization is depicted in Figure 2. The current-voltage curve is depicted in Figure 2 (a). A series resistance of $10 \Omega$ is extracted. A laser threshold current of $40 \mathrm{~mA}$ is achieved (1.5V bias) as shown in Figure 2(b). Laser emission at $1571 \mathrm{~nm}$ is observed as depicted in Figure 2(c). Under higher biases, and higher optical power, mode hopping to the closest lower wavelength supermode is observed. We attribute this mode hopping to a difference of distribution of optical power inside each microring in both mirrors, leading to an effective detuning due to the strong thermo-optical effect in silicon and, thus, to a new supermode condition. A waveguide-coupled output power of $-3 \mathrm{dBm}$ and a $40 \mathrm{~dB}$ side mode suppression ration (SMSR) is obtained.

(a)

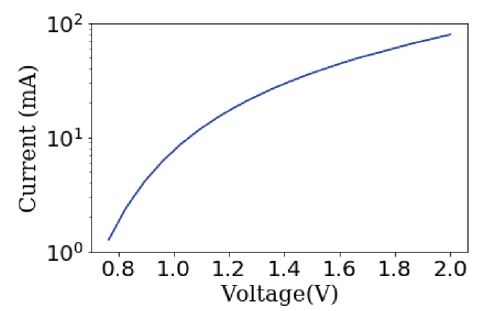

(b)

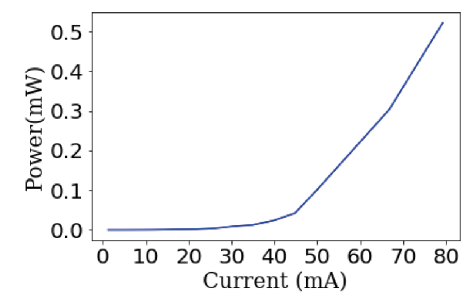

(c)

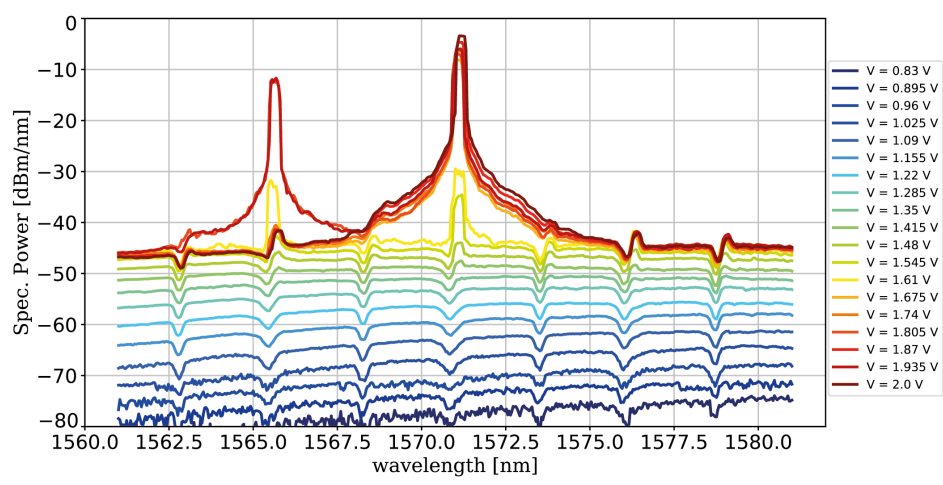

Fig. 2: (a) Current-voltage curve. For high bias voltages, a $10 \Omega$ series resistance is obtained. (b) waveguidecoupled optical power, showing a threshold current of $40 \mathrm{~mA}$. (c) Optical spectrum for various amplifier bias voltages. The laser operates at $1571 \mathrm{~nm}$, with a SMSR of $40 \mathrm{~dB}$

\section{Conclusion}

We demonstrate a novel III-V-on-Si laser based on photonic molecule resonant mirrors. Basic characterization shows a threshold of 40mA with $-3 \mathrm{dBm}$ waveguide-coupled output power at $1571 \mathrm{~nm}$ and a SMSR of 40dB.

\section{Acknowledgment}

This work is supported by Sao Paulo Research Foundation (FAPESP) under funding 2016/23456-1.

\section{References}

1. Z. Zhou, B. Yin, and J. Michel, "On-chip light sources for silicon photonics," Light: Science \& Applications 4 (2015).

2. S. Keyvaninia, G. Roelkens, D. V. Thourhout, C. Jany, M. Lamponi, A. L. Liepvre, F. Lelarge, D. Make, G.-H. Duan, D. Bordel, and J.-M. Fedeli, "Demonstration of a heterogeneously integrated III-V/SOI single wavelength tunable laser," Optics Express 21, 3784-3792 (2013).

3. T. Komljenovic, M. Davenport, J. Hulme, A. Y. Liu, C. T. Santis, A. Spott, S. Srinivasan, E. J. Stanton, C. Zhang, and J. E. Bowers, "Heterogeneous silicon photonic integrated circuits," Journal of Lightwave Technology 34, 20-35 (2016).

4. S. Dhoore, S. Uvin, V. Thourhout, G. Morthier, G. Roelkens, . A. Liu, R. Jones, L. Liao, D. SamaraRubio, D. Rubin, O. Cohen, R. Nicolaescu, M. Paniccia, . D. Liang, A. Fang, D. Oakley, A. Napoleone, D. Chapman, C.-L. Chen, P. Juodawlkis, O. Raday, and J. E. Bowers, "Novel adiabatic tapered couplers for active III-V/SOI devices fabricated through transfer printing," Optics Express 24, 12976-12990 (2016).

5. M. C. M. M. Souza, L. A. M. Barea, A. A. G. V. Zuben, S. Gustavo, and N. C. Frateschi, "Tunable Spectral Engineering of Coupled Silicon Microcavities," 1, 2-3 (2015). 